\title{
When reducing numbers might increase pain
}

Neuropathic pain, or pain that arises from disorders of the nervous system, is a challenge to treat. One of the more common research techniques used to induce neuropathic pain is known as the chronic constriction injury model ${ }^{1}$. The model involves placing a series of loosely tied sutures around the sciatic nerve of a rat. Within about a week after surgery this results in signs of pain in the rat's affected leg, such as licking or biting of the leg, avoiding use of the leg, and limping if the leg is used at all.

Dr. John Foxworthy studies the mechanisms of action of drugs that were used or proposed to be used to treat neuropathic pain. For an upcoming study he proposed using the chronic constriction model on twenty rats that would also receive a drug that was believed to modulate calcium ion conductance. Another twenty rats would be treated with the same drug and undergo the same surgical manipulation but without any constriction of the sciatic nerve. One additional group of five rats would only be treated with the drug. During the IACUC review of Foxworthy's protocol one of the reviewers questioned the need for one of the study groups, suggesting that the surgical procedure without the nerve constriction could be performed on the same rat's other hind limb. That way the number of animals to be used would be significantly reduced, and one leg would serve as a control for the other leg. Foxworthy replied that he would rather use the extra twenty rats, thereby causing less pain to more animals rather than more pain to fewer animals. He also said that even without constricting the sciatic nerve of the contralateral leg, a second surgery on the same animal would confuse the interpretation of his findings.

Do you agree with the opinion of Foxworthy or the IACUC reviewer? Assuming a sample size of $n=20$ is appropriate for each of the two surgical groups, do you believe that $n=5$ is an appropriate number of animals for the non-surgical control group?

1. Bennett, G.J. \& Xie, Y.K. A peripheral mononeuropathy in rat that produces disorders of pain sensation like those seen in man. Pain 33, 87-107 (1988)

\section{RESPONSE}

\section{Request for more information}

\section{Michele M. Bailey, DVM, MRCVS, DACLAM \& Michelle Loh, BVSc}

The IACUC reviewer asks a good question, but we do not feel that the contralateral leg would serve as a good control. Rats receiving surgery on both hind legs would ambulate differently from rats undergoing unilateral ligation or unilateral sham surgery, since the former would not have the option of compensating with an unaffected hind leg. This could interfere with the assessment of pain responses. Previous research indicates that "unilateral constriction of the left common sciatic nerve... gives rise to a marked increase in sensitivity to normally innocuous tactile stimuli in both the nerve-injured as well as the intact contralateral hind paws. Although less in magnitude and duration, surgery alone without nerve constriction also produces a decrease in withdrawal threshold of each of the hind paws" ${ }^{1}$. Accordingly, this study "demonstrated that the contralateral hind paw and either hind paw in sham-operated rats are inappropriate as 'controls."

Additionally, it is unclear which parameters Foxworthy will be monitoring to assess whether the drug treatment is effective. Will he use behavioral, biochemical or other tests? For example, if measuring serum cortisol levels as an indicator of effectiveness, Foxworthy would not be able to distinguish between the drug's effectiveness after nerve constriction and its effectiveness after sham surgery if both legs are on the same animal. If, during publication, reviewers should deem that sham surgery on the contralateral leg is not a scientifically valid control treatment, the experiment might need to be repeated using new and separate groups of rats for nerve constriction and sham surgeries, which would relegate the original group of rats to wasted animals.

Regarding the size of each group, no data have been provided to explain and support the use of 20 animals per experimental group. The investigator should pro- vide justification for the group size. The Guide for the Care and Use of Laboratory Animals states that the number of animals and size of experimental groups should be statistically justified whenever possible ${ }^{2}$. Assuming that the IACUC is given sufficient justification for the group size and finds that 20 rats per group is a valid sample size, it is then unlikely that a control group of 5 rats will reach statistical significance if all groups are assessed according to the same methods. It is important that control groups be of a statistically valid size.

In addition, we feel that the IACUC should query the rationale behind using as controls rats that underwent sham surgery and were treated with the drug and also rats that underwent no surgery and were treated with the drug. If the purpose of the study is to determine a drug's effectiveness in controlling neuropathic pain then a better control might be a single group that undergoes nerve constriction but does not receive the drug treatment. Although such an untreated nerve-constricted group is a less palatable option, it would reduce the number of animals used in total. 